\title{
Application of Chemoinformatics in Asymmetric Catalysis
}
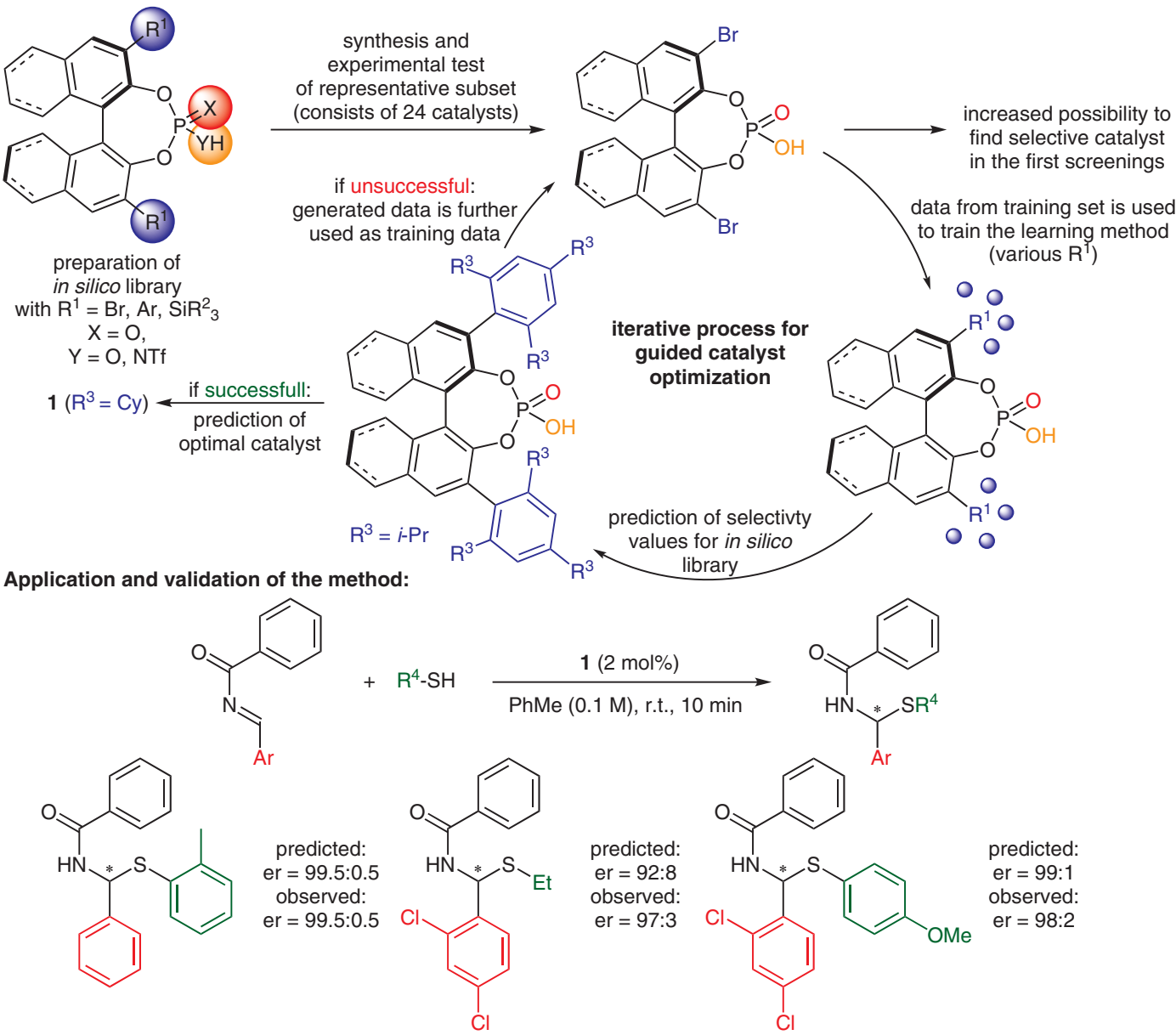

Key words

chiral phosphoric acids

catalyst design

chemoinformatics

acetals

artificial intelligence

Significance: Denmark and co-workers report a computer-driven workflow and a machine-learning method that is capable of predicting the enantioinduction of a range of chiral phosphoric acids in $\mathrm{N}, \mathrm{S}$-acetal formation. The mathematical model is based on a new descriptor introduced by the authors, i.e. the average steric occupancy, which considers the variability of catalyst conformations and describes points in a 3D space in which a given catalyst resides. The mathematical model is iteratively trained with experimental data, leading to a system that can predict selectivities for catalysts that have not been experimentally tested.
Comment: The concept of model-driven method development has far-reaching implications in and beyond the chemical community. However, computer-driven workflows have hitherto not proven capable of predicting experimental results beyond data collected empirically. By describing the population of a catalyst and its conformers in space, a key factor in asymmetric induction, Denmark and co-workers have successfully expanded the utility of such processes, permitting accurate predictions outside the range of the training set. We look forward to the implementation of such a model in systems with greater complexity and in providing solutions to actual problems. 